\title{
La experiencia de Brasil con una Política Exterior Independiente
}

\begin{abstract}
V ícror W A L I s es graduado de Harvard. En la actua. lidad trabaja en Chile para su doctorado en Ciencias Politicas en la Universidad de Columbia (Nucva York), sobre el tema: el impacto de las inversiones extranjeras en la política chilena.
\end{abstract}

Tal como el lenguaje de la carta original de la oEA lo sugiere, es costumbre 'que los países latinoamericanos describan su politica exterior bajo términos de "no intervención" y "autodeterminación". Estos conceptos, sin embargo, están sujetos siempre a cambios drásticos en su significado según quien sea el que los emplee. Por lo que no es demasiado sorprendente verlos invocados tanto por los defensores como por los opositores de las alineaciones tradicionales. Esto mismo vale para el término "independencia". aplicado a la política exterior reciente del Brasil. Aunque sea innegable que el verdadero énfasis hacia la independencia fue un producto de las administraciones de Quadros y Goulart, el lema mismo resultó haber adquirido demasiadia fuerza para poder ser repudiado abiertamente, aun dentro del contexto de una posición pro Estados Unidos tan inaudita como la del régimen Castello Branco. Los voceros de Castello se refugiaron tras el concepto de "interdependencia"; pero al mismo tiempo trataron de sugerir que las innov'aciones del periodo 1961-1964 habian constituido no una aseveración de la identidad brasileña sino más que nada el sometimiento gradual del Brasil a influencias extranjeras ${ }^{1}$.

Descle un comienzo debemos admitir que la idea de independencia es necesariamente un concepto muy relativo para una nación subdesarrollada: hasta que el país logre movilizar sus propios recursos, con

\footnotetext{
* Este trabajo es una versión revisada de un estudio preparado originalmente en los Estados Unidos en 1965. Estoy muy agradecido de Carlos Esteyam, Cleantho de Paiva Leite y Deodato Rivera, por sus comentarios a mi primera versión.

"Theophilo de Andrade, "A Politica Independente e Cuba", O Cruzeiro, mayo 9, 1964, p. 120. Ver también el desmentido del Canciller de Castelo Branco, Vasco Leitão da Cunha, de que la ruptura de su gobicrno con Cuba constituyese un abandono de la independencia. Conferencia de prensa de julio 6, 196:, en Revista Brasileira de Politica Internacional (citada más abajo como $R B P I$ ) vil, 27 (1964), p. 591.
} 
ju propia iniciativa, cualquiera independencia existente se basa sencillamente en su habiliclad para maniobrar ante influencias externas alternantes. En el caso de Brasil bajo Quadros y Goulart, no se puede cliscutir el hecho de que hubo un importante giro, alejándose de la antigua deferencia rendida a Estados Unidos. Quadros mostró un anticipo de este cambio cuando rechazó una invitación a Washingzton durante su viaje como presidente electo en el otoño de $1960^{2}$. El nuevo estilo fue a menudo reafirmado durante los tres años siguientes de diversos modos que consideraremos detalladamente más adelante. Sin embargo, el hecho que verdaderamente marca a este giro con un objetivo esencialmente dirigiclo hacia la independencia, es que desde principios hasta fines del periodo mencionado, los Estados Unidos jamás cesaron de ser el único poder extranjero que podía verdaderamente ejercer influencia sobre la économía brasileña. Esto debido no sólo a su papel como primer mercado para las exportaciones brasileñas, sino también por la magnitud que alcanzaban las inversioncs privadas de Estados Unidos y además los empréstitos gubernamentales obtenidos durante el período de postguerra ${ }^{3}$. Naturalmente, Brasil se vio empujado hacia una posición que le forzaba a contraer obligaciones cada día mayores; al propio tiempo, el peso mismo de esta relación estimulaba una búsqueda de posibilidades de desarrollo alternativas.

El esfuerzo de Brasil para lograr una política exterior independiente no fue algo sin precedentes dentro de los países de América Latina. Su singularidad fue que surgiera tạn de súbito como to hizo, sin ser el resultado de una revolución social previa ${ }^{4}$. Para explicarse esta peculiaridad -que a su vez deternina las limitaciones en el grado cle independencia efectivamente realizado- es necesario considerar no tan sólo la naturaleza del desarrollo económico de Brasil y sus reĩa-

2Hispenic American Report (citada más abaja como HAR) 13 (1960), p. 836.

$241 \%$ de los ingresos de cxportaciones del Brasil provinieron de Estados Unidos en 1961, $38 \%$ en 1963. Publicación del Departamento de Comercio de Estados Unidos, Overseas Business Reports 63-70 (mano; 1963), p. 5; 64-75 (junio, 1964), p. 22. La inversión total de Estados Únidos en Brasil se establece por esta misma fuente, en 1962, en us $p$ 1.088 miliones (junio 1964, p. 20). La deuda total de Brasil con Estados Linidos en 1961 era de es\$ 1.759 millones, con una deuda adicional de us\$ 1.100 millones en el Fondo Monctario Internacional (Neiv Yark Times, 18 de mayo de .1961 , p. 6). El presupuesto federal anual de Brasil en esta fecha ascendía aproximadamentc a us\$ 1.500 millones (misma fuenie).

IEl caso de México, que se ha mantenido aparte de cuslquier alianza militar de postgucra con Estados Unidos (J. Lloyd Miccham, The United Slates and Inter-American Security, 1889. 1960, Austin, Texas, 1961 , p. 35) $\mathrm{y}$ - que actualmente es el único miembro de la ops que mantiene lazos aficiales con Cuba, debe recordarse como contraste. Las desviaciones de Aryentina $y$ Boliyia durante la segimda guerra mundial no son licitamente comparables, ya que la influencia de Estados Unidos en esos paises antes de la guerra era relativamente pequeña. Otrosizasos de posiciones independientes ante los Estados Unidos están más bien asociados ya sea con revolución o con tradiciones anteriores al periodo postguerra. En todo caso, cl asunto merece mayor estudio. 
Victor Wallis / La experiencia de Brasil con una Bolitica Exterior Independiente

ciones con los Estados Unidos, sino también los grandes cambios que estaban sucediendo en otros lugares del mundo a fines de la década de 1950 y el significado especial que revestían para Brasil. Aunque la política exterior independiente derivó su nombre dentro de un tenor de dominación de Estados Unidos, distaba mucho de basarse exclusivamente en sentimientos antinorteamericanos. Mirado en retrospectiva, esto se ve claramente ya que, de los tres cambios en política exterior más importantes iniciados por Quadros y Goulart -1) relaciones diplomáticas con países del bloque soviético, 2) mayores contactos con naciones subdesarrolladas del hemisferio oriental, y 3) oposición a la presión de Estados Unidos para lograr sanciones contra Cuba- solamente el cambio de actitud hacia Cuba fue inequírocamente repudiado por Castello Branco.

Dos de las condiciones más importantes que favorecieron la política exterior independiente se establecieron solamente unos años antes de la elección de Quadros. Una condición fuc el relajamiento parcial de la tensión existente entre Estados Unidos y la Unión Soviética; lá otra, el surgimiento desde el colonialismo de las nuevas naciones africanas -21 naciones emergieron entre 1956 y $1960^{\circ}$. Aunque cada uno de estos acontecimientos trajo consigo sus propias oportunidades y problemas, ya fuera en la esfera económica o la política, eran similares en ofrecer a los líderes del Brasil una base mucho más aceptable que la anterior para iniciar tentativas hacia una orientación distanciadora del rígido molde bloque hemisférico para los asuntos internacionales. Aunque estos cambios no explican todos los aspectos de la línea independiente, por lo menos forman gran parte de la trama dentro de la cual se debatió ésta.

La détente Estados Unidos-Unión Soviética, incorporada en las reuniones de Eisenhower y Khruschev de agosto de 1959, tuvo para Brasil la significación de ser el punto álgido de una larga serie de directas invitaciones soviéticas tendientes a relaciones más unidas ${ }^{5}$. La administración de Kubitschek, hasta entonces, había rechazado estas proposiciones y sólo las había mencionado en público como un medio de asegurar mejores arreglos financieros con los Estados Uni-

¿La conexión directa se ve claramente en una declaración del Canciller Horácio Lafer en el sentido de que estas reuniones forzarian al Brasil a reconsiderar su politica hacia 12 Unión Soviética (Veze York Times, agosto 5, 1959, p. 5). 
dos$^{6}$. Si acaso Kubitschek hubiese llegado más lejos que esto sin la détente es, por supuesto, una pregunta teórica; y la importancia del ejemplo de Estados Unidos no debe permitir que se esconda la presión de consideraciones estrictamente económicas, sobre todo la enorme baja de entradas obtenidas por las exportaciones de café y el suministro insuficiente de petróleo brasileño ${ }^{\tau}$. A pesar de todo, sólo en diciembre de 1959 fue firmado un acuerdo comercial importantes. Kubitschek mismo continuó rechazando la idea de relaciones diplomáticas, pero el deseo de conseguir relaciones comerciales más amplias comenzaba a adquirir tal importancia dentro de los círculos políticos, incluso antes de 1959, que el argumento por un intercambio diplomático total adquirió fuerza, aunque sólo fuese con el fin de asegurar sobre una base más estable los beneficios del intercambio comercial'. En cuanto a las implicaciones políticas de esta medida, el profesor Hélio Jaguaribe había indicado ya en 1958 el significado directo que para Brasil tenían precisamente los mismos cambios clentro del mundo comunista que posibilitaban una clétente Estaclos Unidos-Unión Soviética ${ }^{10}$. Señalando la "ruptura del monolito comunista", la derrota de los particlos comunistas de Europa Occidental y la ausencia de influencia del Partido Comunista en los movimientos de independencia - en Africa, él predijo que habría un triunfo generalizado del nacionalismo sobre el comunismo en cuanto a ideologías y consicleró inconrbible que la Unión Soviética iniciase una nueva guerra mundial. Su conclusión, bajo las circunstançias del momento, fue que el concepto prevaleciente de "seguridad nacional" había sido transformado en "un mecanismo de la subordinación del poder nacional brasileño " los intereses estratégicos de los Estados Unidos". Considerando támbién los atspectos coloniales de la economía de Brasil, argumentó en general que "las amenazas más cercanas à nuestra capacidad de autode-

Iniciativas soviéticas para restabjecer relaciones (que habian existido entre 1945 y 1947) fucron destacadas en el Neve York Times ja en julio 18, 1956. Una tipica mención de Kubitschek ocurre durante su discurso del 26 de novicmbre de 1958 en la Academin de Guerra, en $R B P I$, II, 5 (1959), p. 148. Vargas tuvo un temprano interés en el intercambio comercial sovictico, pero éste no se materializó antes de su muerte (Entrevista con Clcantho de Paiva Leite, consejero en asuntos exteriores de Vargas, 1951-54. Santiago, Chile, marzo de 1967).

"Clifford Frank Owen, "U. S, and Sovict Relations with Underdeveloped Countries: Latin America - A Case Study", Inter-American Economic Alfairs 14 (Winter, 1950), p. 100.

sNeto York Times, dicicmbre 12, 1959, p. 8. Un pequeño negocio de intercambio fue electuado en novicmbre de 1958 entre Petrobras y la agencia soviética exportadara de petróleo; pero no se cstablecieron rolaciones comerciales oficiales hasta novicmbre de 1959 por el gobierno de Brasil (Owen, n. 7, p. 98).

"Amilca: Alencastre, $O$ Brasil e as Relasōes com o Lesle e a U.R.S.S. (Río de Janeiro, 1959), p. 16 .

10Ver sección 3 de Jaguaribe, $O$ Nacionalismo na Alualidade Brasileira (Río de Janciro, 1958), un póroducto del Instituto Superior de Estudos Brasileiros, Mlinisterio de Educación. Sabre la significancia de esta obra, ver Frank Bonilla, "A National Idcology for Development: Brazil", en Kalman H. Silvert, ed., Expectant Pcoples: Nationalism and Development (New York, 1963), y. 236. 
terminación vienen mucho más de parte de Estados Unidos que desde la Unión Soviética"11.

Sin embargo, como una defensa específica de las relaciones diplomáticas soviético-brasileñas, tal vez la declaración más influyente fue la de Oswaldo Aranha, por lango tiempo compañero político de Vargas y su Ministro de Relaciones Exteriores durante la segunda guerra mundial. Los argumentos ${ }^{12}$ de Aranha se basaron en: 19, el deseo de Brasil de llegar a ser uno de los diez grandes poderes, $2 \circ$, la no relación entre idleología y reconocimiento diplomático, 39, relaciones existentes con Polonia y Checoslovaquia, 4\%, ventajas comerciales, $5^{\circ}$, el ejemplo de los Estados Unidos, y $6^{\circ}$, la necesidad de que Brasil participase en decisiones globales. Sería muy difícil determinar la importancia relativa de cada uno de estos argumentos. En todo caso, es notable que todas estas razones, excepto el argumentó sobre el ejemplo de Estados Unidos y algunos aspectos de su argumento comercial, bien podrian haber sido utilizados - por otros tanto como por Aranhapara apoyar el reconocimiento de la República Popular China. Jaguaribe en realidad defendió este paso, pero el hecho de que no haya sido ampliamente discutido dentro de los círculos políticos sugiere que por muoho que Estados Unidos se preocupase pór las implicancias del acercamiento soviético-brasileño, seguía siendo la détente Estados Unidos-Unión Soviética lo que permitía este acercamiento en esa etapa.

El sưrgimiento de las naciones africanas trajo consigo una amenaza potencial para Brasil -como también para algunos otros países latinoamericanos- desde el punto de vista económico. Esta amenaza consistía en la creciente competencia que enfrentarían los productos primarios de América Latina dentro del mercado mundial. Para Brasil, cuyros ingresos en moneda extranjera el año 1960 seguían dependiendo en un $56 \%$ del cafétis, difícilmente podía ser una idea reconfortante el hecho que, habiendo aumentado la producción anual mundial de café en un $90 \%$ desde el periodo $1950-55$, constituyera el incremento africano un $105 \%^{14}$. Esta situación solamente podia acelerar las ya enormes pérdidas de ganancias del Brasil, aunque los efectos igualmente dañinos que a la larga tendría sobre los productores africanos de café podría proveer un buen punto de partida para negociaciones. por otro lado, sin embargo, desde una posición en búsqueda de una disminución de la dependencia exclusiva en el café, la independencia

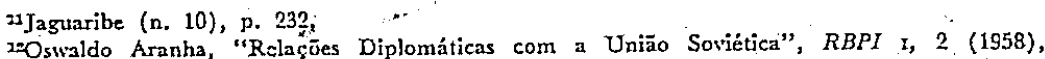
pp. 20-26.

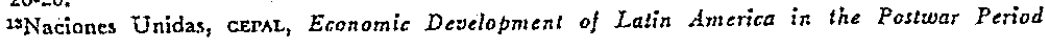
(New York, 1964), p. 145.

"Jarbas Maranhão, Brasil-Airica: Um Mesmo Caminho (São Paulo, 1962), D. 16. 
total de Africa podía sólo beneficiar a Brasil en el sentido 1) de hacer posible por primera vez. un aumento de los costos sumamente bajos de materias primas que gozaban las industrias de los poderes coloniales, así quitando por lo menos un obstáculo a la potencial competencia de productos manufacturados brasileños dentro del mercado mundiali5, y 2) abrir a la vez el propio mercado africano a los productos brasileños ${ }^{16}$. La discusión cle estas posibilidades estuvo siempre compenetrada de una nota de escepticismo, sin embargo; por un lado, sin cluda, debido al bajo poder comprador de la población africana; por otro lado, debido al éxito evidente de los países pertenecientes al Mercado Común Europeo en establecer relaciones especiales con Africa ${ }^{17}$. Ambas condiciones eventualmente deberían ser superadas, pero el tipo de organización que esto requeriría distaba mucho de poder prometer beneficio a corto plazo para el Brasil.

O. sea, que la importancia más inmediata que la independencia de Africa ofreció a Brasil se basó no tanto en los proyectos específicos que aparecieron tangibles, sino más bien en la oportunidad que bien se aprovechó hacia la formación de una ideología nacional positiva. Hasta ese momento, Brasil no había lógraclo poder hacer distinciones entre su posición internacional y la clel Hemisferio Occidental, vale decir -en efecto- la de los Estados Unidos. Al exigir relaciones con la Unión Soviética, los jefes brasileños explícitamente omitieron toda consideración icleológica, excepto un deseo abstracto por el status de gran poder mundial y tal vez un vago sentido de compromiso hạcia la obtención de la paz por medio del entendimiento. Al emerger Africa, sin embar:go, Brasil puclo sumar a su proyección internacional un énfasis generalizado a sus intenciones de desarrollo, combinado con referencias específicas a caracteristicas nacionales propias del país. En el terreno de la geopolítica, Brasil pudo considerar la posibilidad de suplementar entidades ya existentes tales como "Atlántico Norte" - "Hemisferio Occidental" con una nueva formación "Atlántico Sur", la cual por lo menos incluiría a los países de la costa sureste de América a la vez que Africa Occidental. Tras esta idea, tal como fue elaborada por Jaguaribe, yacía la experiencia con bases militares norteamericanas en Brasil durante la segunda guerra mundial. Durante cualquier eventual conflicto Oriente-Occidente, tales bases, lejos de contribuir a la seguridad del Brasil, lo dejaban vulnerable al ataque:

\footnotetext{
"Este punto seria nencionado por Quadros en su articu'o, "Brazil's New Foreign Policy", Foreign Alfairs 40 (1961), p. 25.

ItAdo:fo Justo Bezerra de Menezes, Asia, Africa, e a Politica Independente do Prasil (Río de Janciro, 1961), p. 110.

17Estanislau Fischlowits, "Stubsidio para a 'Doutrina Africana' do Brasil", RBPI 1II, 9 (1960), p. 89.
} 
una perspectiva que los adelantos tecnológicos hacían mucho más próxima que antes. Contando con una alianza sur-atlántica indepencliente, ninguna imposición de ese orùen tendría que ser aceptada, y Brasil podría ser capaz de determinar cualquier política defensiva libre de los grandes poderes en conflicto1s.

Por utópica que fuese esta idea, en la medida que se basase en las capacidades militares de Argentina y Brasil, la independencia que esperaba lograr poclía ser aclelantada de un modo general (más económico e ideológico que militar) por alguna forma de ajuste con las ideologías existentes de no alineación o neutralismo. La forma precisa que este ajuste tomaría seguiría siendo materia de discusión, aunque la tendencia más difundida en Brasil era la máxima disminución de cualquier posibilidad de eventual ruptura con la ideologia occidental. En cuanto a los nuevos puntos de énfasis sugericlos, los más populares aparentemente fueron el lazo étnico de Brasil con Africa, la relativamente amplia integración del elemento africano a la cultura nacional brasileña, y finalmente, la posibiliclad de que, dados su tamaño, póblación y experiencia, Brasil estaría en posición de suministrar alguna forma de liclerato para Africa -incluyendo las formas de asistencia menos costosas, tales como entrenamiento universitario- que no estarían teñidas con imperialismo, supremacía blanca, o consideraciones de guerra fria ${ }^{19}$. Toclos estos puntos de énfasis, aunque eran compatibles hasta con un molde radicalmente cambiado deI clesarrollo interno de Brasil, no presuponían nada por el estilo, e incluso muy fácilmente podian ser usados con objetivos propagandísticos sin cambio interno alguno.

Pero si acaso la existencia de los nuevos estados africanos, junto a la délente Estados Unidos-Unión Soviética, podía alentar incluso a un gobierno de base conservaclora hacia una reorientación de su política externa, otros cambios, a la larga mucho más prodigiosos, determinaron los problemas específicos que tal reorientación habría de confrontar. La posibilidad africana desde un comienzo fue dañada por el papel de Portugal, país que aún mantenia a tres territorios bajo la férula colonialista. Portugal mantuvo una posición intransigente en contra de su independencia y ejerció una continua presión sobre Brasil por medio de poderosos grupos comerciales de Rio de

${ }^{28} J a g u a r i b e$ (n. 10), pp. 291-95. Es curioso notar que al contrario de los futuros voceros de la linea cxterior independiente, Jaguaribe propone aqui que Sudamérica tenga sus propias armas nucleares.

${ }^{20}$ Ver especialmente Adolío Justo Bezcrra de Menezes, $O$ Brasil e o Mundo Asio-Africano, 1* edición (Rio de Janciro, 1956), pp. 308 y 319, sobre la habilidad de Brasil para divulgar lo mejor del legadó occidental por medio de su ejemplar mezcla racial; también Amilcar Alencastre, Ostaldo Aranha, o Aundo Afro-Asiatico e a Paz (Rio de Janeiro, 1961), p. 79, sobre el ancestro "brasilcüo" de ciertos lideres africanos. 
Janeiro y São Pauloº. O sea, que hubo movimientos que Brasil podía celebrar en el resto de Africa $y$, sin embargo, acoger confusamente cuando surgían clentro de las colonias portuguesas. Este dilema se acentuó cuando se inició el movimiento de independencia de Angola a fines de la década pasada; pero el voto de Brasil en las Naciones Unidas invariablemente continuó dentro de las filas de los poderes coloniales, incluso durante resoluciones puramente verbales respecto a Africa, hasta fines de 1960"1.

El mayor dilema de todos, sin emljargo, Lue el que ofreció la Revolución Cubana. Habienclo triunfado dos años antes de la ascensión de Quadros a la Presiclencia, aseguró que no se produjera un florecimiento prematuro de visiones funcladas exclusivamente en el acercamiento Estados Unidos-Unión Soviética o en un apoyo africano ambiguamente solicitado. El desatío urgente que significó para los Estados Unidos, junto a su gran proximidad psicológica al Brasil, si se compara con cualesquiera de los desarrollos africanos, llevó inevitablemente al Brasil a tomar una posición clara y definicla. Pero al mismo tiempo, el problema de Cuba significó que si una politica exterior independiente habría de existir, su defensa sostenida no sólo estaría condicionada a una fórmula jurídica posiblemente feliz, sino también a desenvolvimientos sobre los cuales el gobierno brasileño no tendría control alguno; vale decir, el curso recorriclo por las relaciones Estados Unidos-Cuba y el endurecimiento de la oposición conservadora influida por los Estaclos Uniclos dentro del Brasil.

El problema resultante al tratar de explicar el alcance total de la política exterior inclependiente es que ésta fue mucho más allá de ser una mera respuesta a las oportunidades y desafíos presentados por la Unión Soviética y Africa. Debiclo a su naturaleza misma, la línea independiente señaló un punto de cambio de dirección tanto como una expansión de horizontes. Para comprencer este aspecto más fundamental, es necesario consiclerar los problemas del desarrollo económico de Brasil. Ya hemos señalado el fenómeno de la deterioración de los términos de intercambio, los que desde alrededor de 1955 en adelante afectaron a casi todas las exportaciones de productos primarios. Como una reacción inmediata, esta situación naturalmente sugirió la búsqueda de mercados alternativos que ofreciesen mejores

\footnotetext{
so José Honúrio Rodrigues, Brasil e Africa: Outro Horizonte, 2* edición (Río de Janeiro, 1964), p. 361 .

"Maria, Y. Leite Linhares, "Brazilian Forcign Policy and Africa", The World Today 18 (1962), p. 534 .
} 
condiciones, lo que a su vez señaló la necesidad de intercambiar relaciones diplomáticas con los potenciales compradores y a la vez unir lazos con los posibles rivales. Pero, visto a más largo plazo, lo realmente necesario era un cambio en la composición misma de las exportaciones, desechando los productos no elaborados y fomentando los manufacturados. El interés brasileño por esta vía de acción se reconoce claramente en sus deliberaciones sobre Africa, pero en cuanto a la real habiliclad para efectuar tal programa, la economía de Brasil fue una víctima, hasta cierto punto, de su desarrollo anterior. Por necesidad durante los años de crisis económica de 1930, pero en gran parte por libre elección durante la década de 1950, la inclustria montada por Brasil estaba casi exclusivamente ajustada para satisfacer las demandas inmediatas del mercado local. Aparte de las características restringidas de este mismo mercado, este enfoque trajo consigo por lo menos dos complicaciones: 1) Al dedicar mayor energía y atención a la clemanda inmediata que a los recursos existentes, se posibilitó el establecimiento de inclustrias que por largo e indefinido tiempo estarian sujetas a su dependencia en la jmportación para suministros o componentes esenciales. Como consecuencia, Brasil estaría mucho menos capacitado que anteriormente para apelar a la restricción de importaciones como una medida correctiva a los déficit en el mercado internacional. 2) Al mismo tiempo que aumentaba la dependencia en determinadas importaciones, los precios de las exportaciones primarias disminuian $y$ continuarian ejerciendo el peso de su influencia sobre la balanza de pagos ${ }^{22}$.

La crisis que asomaba implícita en este modo de desarrollo, subyacia en todos los aumentos espectaculares de producción efectuados durante el periodo de Kubitschek (1956-51). El flujo de préstamos extranjeros sólo podía calmar momentáneamente lo que, a la larga, agravaba. En cuanto a inversiones clirectas de capital extranjero, hubo cn efecto un vuelco en la situación usual de mayores remesas anuales de utiliclades que entradas anuales de nuevo capital23, pero únicamente a un duro precio futuro, porque además de indicar un eventual

-Este ansilisis se basa en Celso Furtado, Dinlética do Desenvolvimento (Rio de Janciro, 1964), pp. 99, 114; y el Informe del Secretario General (Raúl Prebisch) sobre la Conierencia de Comercio y Desarrollo, $\mathrm{NU}$ ( $\mathrm{New}$ York, 1964), passim. Los ingresos totales de exportaciones bajaron desde un promedio anual de us 5 1.540 millones en cl periodo 1951-55 a us\$ 1.214 millones en 1962 (Niaciones Unidas, Economic Survey of Latin America, 1964, New York, 1966, p 77); el precio del café bajó de us $\$ 70$ por saco en 1953 a us $\$ 39$ el saco en 1962 (Visäo, abril 12, 1963, p. 20).

monilla (n, 10), p. 247. Werner Bacr y Mario Simonsen, en su ensayo, "American Capital and Braxilian Nationalism", Yale Review (invierno, 1964), p. 193, cometen un error al decir que la balanza durante el pęriodo Ǩubitschek fue típica. Para cifras anteriores ver Joint BrazilU.S. Economic Deiclopment Commission, The Development of Braxil (Washington, 1954), desde p. 55; para cilras' más recientes, ver ediciones de agosto de U.S. Department of Commerir. Survey of Current Business, desde 1962. 
aumento en las remesas al extranjero, las nuevas inversiones extranjeras traían consigo una natural tendencia a exagerar aún inás la ya excesiva concentración de producción de bienes de consumo para el mercado local24. A pesar de los efectos dañinos de ambas consecuencias sobre la balanza de pagos, Kubitsch́ek estimuló el proceso al mantener los subsidios cambiarios para importaciones de inversionistas extranjeros que habian sido instituidos en 195525 . II resultado fue una tendencia permanentemente inflacionaria, la cual Kubitschek exacerbó con su fuerte financiamiento dé déficit de proyectos tanto productivos como improductivós.

Como respuesta a esta situación, surgió un difundido sentimiento nacionalista enfocado en el problema del desarrollo económico, abogando especificaménte por una salida dèl molde ya exisțente de contracción de obligaciones extranjeras cada vez más desmesuradas como una condición necesaria al futuro crecimiento del Brasilac. Este sentimiento nacionalista no incorporaba una concepción uniforme de las alternativas económicas, aunque en general favorecia, por lo muy menos, una reducción de los privilegios extranjeros y del poder también extranjero para tomar decişiones ${ }^{27}$; pero tưvo una indudable repercusión dentro del escenario internacional, a saber, que Brasil no debía recibir órdenes de nadie y debía derender el derecho de otras naciones para determinar sus propias rutas de desarrollo. Aunque esta posición estaba igualmente disponible para nacionalistas de otros paises, la fuerza especial que ganó en Brasil alrededor de 1960 debió algo a la velocidad, al repentino aumento de inversión extranjera, y la inestabilidad financiera que caracterizaron al período más reciente del crecimiento económico brasileño.

Pero por mucho que tales consideraciones señalaron la necesidad de una politica exterior independiente, difieren del deshielo de la

2Sobre los motivos de esta concentración, ver Lincoln Gordon y Engelbert L. Grommers, United States Mianufacturing Investment in Brazil, 1946-1960 (Cambridge, Mass., 1962), p. 148, y Cleantho de Paiva Leite, "Brasilian Development: One Problem and Tivo Banks", InterAmcrican Economic Affairs it (verano, 1960), p. 7.

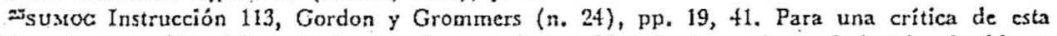
política de extensión del control extranjero $y$ destrucción de la naciente industria de bienes de capital de Brasil, ver Sérgio Míagalhães, Problemas do Desinuolvimenio Econômico (Río de Janeiro, 1960), p. 35 .

2rVer el cstudio de Bonilla y las publicaciones del ISEB (n. 10); también la Revista Brasiliense, especialmente Elias Chaves Neto, "O Presidente Kubitschek e o Fundo Aonetário Internacional" (julio-agosto, 1959, $\mathrm{pp}$. 1-8) y el ataque de " $\mathrm{xxx}$ " a Jaguaribe, destacando la falta de oposición de éstc al capital privado cxtranjero ("Nacionalismo e Problemas Brasileiros", cnero-febrero, 1959, pp. 35-50); también las observaciones de John J. Johnson, The Mlilitary and Society in Latin America (Stanford, 1964), p. 222, sobre el vuclco del nacionalismo después de 1955, rumbo que abandonaba ahora su primaria concentración en las fucras armadas.

zisubre ef frecuente prejuicio $y$ corrupeión de este nacionalismo, asociado con su énfasis dirigido exclusivamente hacia el aspecto anti-listados Unidos, ver Tinothy" F. Flarding, "Revalution Tomorrow: The Fuilure of the Leit in Brazil", Studics on the Left 4 (otoño, 19G4), p. 37. 
guerra fría y el problema de . Ifrical en el sentido de que, lejos de estimular una reorientación : le par!: de los conservadores, implicaron un ataque a la posición conscrvadoma nisma. Mientras que la expansión cle mercaclos y los acuerdos con los competidores podían ser $\checkmark$ istos como suplementarios a activiclucles ; relaciones ya existentes, una crítica general del clesarollo reciente del Brasil tendría que teñir de dudas estas actividades y rclaciones. Los voceros de tal crítica, además, tendrían que defender el derecho de Cuba de planear su propio camino sin interferencias hustiles: pocición que, por no decir más, clistaría de servir el interés clirecto de los principales clefensores del conservantisno. Esto no significa, sin embarzo, que la perspectiva de un nacionalismo económico no polía ya ser utilizada por un gobierno que no tenía la menor intención de aplicar su crítica en el frente propio. AI contrario, podia servir para tres objetivos desde tal punto de vista, todos cle posible importancia politica. 1) Al señalar el desarrollo cubano como lezítima aunque incleseable alternativa, el gobierno polía posar una amena\%a efectiva en su campaña pro paliativos externos, tales como nuevas inversiones, mayores préstamos, o amortizaciones diferidas. 2) Al defencler a Cuba contra sanciones internacionales, el gobierno poclía conquistar apoyo provisorio de algunos de sus críticos nacionalistas $y$ cłe izquierda sin satisfacer sus demandas económicas, mientras que, al mismo tiempo, 3) añadía efectividad a sus iniciativas diplomáticas en el resto clel mundo.

De más está clecirlo, el único tipo cle régimen que automáticamente podría ser exonerado del cargo de así "usar" el nacionalismo económico para fines contrarios, scría el régimen que estuviese comprometiclo a efectuar un amplio programa de expropiación, acompañado por cambios estructurales tan completos como para alterar la distribución de riquezas existentes. En la ausencia de tal programa, se da fácilmente cabicla a la especulación sobre las intenciones futuras de quienes utilicen lemas nacionalistas. ¿Planearian o no una eventual traclucción de tales lemas a una acción clentro dẹl pais? Aun'que sea: necesario referirnos a las variables posibles en tales especulaciones, podemos,captar las bases más importantes de la política efectivamente seguida si nos concentramos en factores con los cuales determinados políticos tendrian que laborar, cualquiera fuese su convicción personal. En este sentido, será útil aproximarse a la perspectiva del gobierno brasileño durante la etapa de 1961-1964, revisando sumariamente a) los primeros pasos en dirección hacia una línea independiente tomados hasta por Kubitschek, y b) el sentido en el cual las agrupaciones -políticas de 1960 - podrían impulsar a un tipo de campaña tal como. la realizada por Quadros. 


\section{ESTUDIOS I N TER NAG IONALE S}

Las principales declaraciones ideológicas de Kubitschekententro del: campo de la política exterior estaban conectadas con la Operaçäo Panamericana, el programa de asistencia de Estados Unidos y lazos más fuertes que él propuso después de las demostraciones de odio hacia el Vicepresidente Nixon durante su gira sudamericana en 1958. Al proponer el programa de la OPA, Kubitschek celebró tanto a la Doctrina Monroe como al anticomunismo 28 . A pesar de esto, él aseveró que el sentimiento de agravio popular era auténtico en Latinoamérica, $y$ al mismo tiempo explícitamente reconoció las recientes iniciativas de asistencia extranjera de la Unión Soviética? ${ }^{29}$. Aunque él mismo no proponia disminución alguna de la influencia de Estados Unidos sobre América Latina, Kubitschek pudo, por lo menos, así confrontar a los Estados Unidos con un abierto y casi oficial desafío de que tal vez tal proceso podria en un futuro bastante próximo ser realizado bajo otros auspicios ${ }^{30}$. En el campo de la economía política, correspondientemente, aunque Kubitschek fomentaba una participación de capitales extranjeros cada vez mayor en la industria del pais, al mismo tiempo logró no utilizar los servicios del Fondo Monetario Internacional cuando las exizyencias fiscales de éste no coincidían con sus propios planes ${ }^{31}$. En otros campos, su plan de acción en una $u$ otra ocasión incluyó 1) adhesión a la posición del Mariscal Lott y de elementos del PSD (Partido Social Demócrata) que, junto a la PTB (Partido Trabalhista Brasileiro), opusiesen resistencia a la participación extranjera en la producción cle petróleo32, 2) resistencia temporal ante las demandas de Estados Unidos en cuanto a derechos sobre los minerales radiactivos ${ }^{33}$; 3) la venta de azúcar a China ${ }^{34}$, 4) una proposición para actuar como mediador en la disputa Estados UnidosCuba de $1960^{35}$, y 5 ) sofocación de un atentado dentro del Ministerio del. Exterior, también en 1960, para negar visas a viajeros desde $\mathrm{Cuba}^{3 \mathrm{in}}$.

25iscurso del 29 de octubre de 1958, en la Facultad de Leyes de la Universidad Católica de Rio de Janeiro, RBPI It, 5 (1959), espec., p. 140.

गJuscelino Kubitschek de Oliveira, A Marcha do Ananherer (Säo Paulo, 1962), p. 179; también cita anterior (n. 6).

${ }^{30}$ Discurso Kubitschek (n. 28), p. 1+2. Para poner de relieve las novedades de la opa, ver en la misma Revista ur, 9 (1960), Cclso A. de Souza c Silva, "opa: Antecedentes e Perspectivas", p. 47; sobre lo inadecuado del programa de desarrollo, ver Gándido Antönio Míendes de Almeida, "Política Externa e Nação em Processo", Tempo Brasileiro I (septicmbre, 1962), desde p: 46.

a1Leite (n. 24), p. 16. Se debe mencionar, sin embargo, que a fines de su administración una vez más obtuvo un préstamo del FM, $H A R 13$ (1960), p. 348.

asH.AR 10 (1957), p. 326 (PSo-Parlido Social Demócrata, el partido de Kubitschck; PTB Partido Trabalhista Brasileiro, el partido de Goulart. El tercer partido fue el conservador udN Uniäo Democrática Nacional).

saRescisión del acuerdo, HAR 9 (1956), p. 401; reaceptàción, HAR 9 (1956), p.:605.

aHAR 11 (1958), p. 288.

${ }^{5} H A R 13$ (1960), p. 211.

sopaulo Motta Linia, "JK em Face do Enigma do Itamarati", Revisla Brasiliense (sept." oct., 1960), p. 130 . 
Por muy limitadlas que aparezcan estas decisiones vistas en forma retrospectiva, no dejaron de tener significancia en su época. En especial, el mero hecho de que en sus peticiones de ayuda a Estados Unidos Kubitschek resistía las exigencias hacia una ortodoxia financiera cuando no le convenian, hacia muy difícil que alguien se le opusiese principalmente en nombre de esta ortodoxia y el pago de la deuda, sin ser tildado como un ser absolutamente sometido a los intereses norteamericanos. Ya que el PSD de Kubitschek, con Lott como candidato presidencial en 1950, manturo su coalición con el oficialmente nacionalista PTB dirigiclo por el Vicepresidente Goulart (quien se presentaba a una reelección), el curso lógico para Quadros, como candidato unN (Uniäo Democrálica Nacional), queriendo extender su área de apoyo más allá que la abarcada por sus partidarios en cuanto a programa económico y a sus ataques a la corrupción, fue de apropiarse los reclamos nacionalistas de su oposición ${ }^{3 i}$. La imagen de integridad personal que Quadros trazaba durante la campaña, concordaba perfectamente con esta lógica, porque dificilmente cabia mejor expresión de tal imagen que el más completo desclén por las prácticas establecidas. Al transmitir este desclén a la esfera de la politica exterior, Quadros fue, sin lugar a duclas, ayudado por el hecho de que el Partido Comunista continuaba con su apoyo usual al PTn, o sea, a Lott, y que Lott, deseando renegar de este apoyo, fue obligado a debilitar su nacionalismo por ejemplos tales como su posición abiertamente contraria a las relaciones con la Unión Soviética ${ }^{38}$. Quacros, por su parte, fue mucho más lejos de la posición nacionalista minima del gobierno. El no sólo apoyó el dominio federal de la industria petrolifera (desdiciendo asi una declaración anterior) ${ }^{3 n}$, sino que también apoyó las relaciones diplomáticas con la Unión Soviética, visitó Cuba durante su campaña (marzo, 1960), y hábló en favor de la reforma agraria cubanato.

La conducta de los dos candidatos principales durante tas elecciones de 1960 señala así una práctica general hacia la identificación de cada candidato con precisamente el programa opuesto al presentaclo por sus partidarios más conocidos y bien organizados. La ordenación

srEsta base para el nacionalismo de Quadros se sugiere en parte como una prolongación al nivel nacional de su sintesis anterior de reclamos dẹ muy diversos estratos sociales a nivel estatal, donde la campaña anticorrupción habia resultado suficiente por sí sola. Ver Oliveiros S. Ferreira, "A Crise do Poder do Sistema c as Eleiçōes Paulistas de 1962", Revista Brasileira de Estudos Politicos No 16 (enero, 1964), desde p. 184. Sin embargo, por otro lado, Lloyd A. Free encontró por medio de su cncuesta de la opinión pública, Sonte International Implications of the Political Psychology of Brazilians (Princeton, 1961), p. 38, que la preocupación general de] público respecto a la política exteriọ cra relativamente cscasa.

sLuis Carlos Prestes, Por Que os Comunistas Apoiam Loll e Jango (Río de Janeiro, 1960), reconoce esta posición de Lott pero reasegura ci apoyo a Lott de rodos modos, p. 14.

${ }^{2}$ La prinicra posición, $H A R$ 10 (1957), p. 326; reversión, $H A R 13$ (1960), p. 649 .

${ }^{\circ} H A R 12$ (1959), p. 405; HAR 13 (1960), p. 211. 
de las fuemas en torno a la clección, sugiere que la tendencia a 1$) \mathrm{mi}$ -

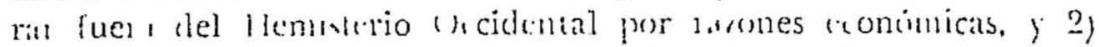
efectuar declaraciones anti-Estados Unidos por motivos políticos, se hacía cada vez más lutil, incluso para grupos en gran parte responsables del aumento del poder conómico de los Fstados I'nidus dentro del Brasil.

\section{IV}

La politica exterior independiente se prolonzó casi sin cambios en su formulación esencial durante los siete meses de presiclencia de Quadros y los dos años; medio de Goulart. Quadros durante su breve perión completó la difícil tarea de iniciación, formulando las principales líneas ideológicas de todo el periodo mencionado y, además, efectuando todos los pasos diplomaticos decisivos, salvo el intercambio mismo de embajadores con la Unión Soriética. Incluso respecto a Cuba, su firme posición contraria a la intervención de Estados Uniclos fue ciertamente tan fuerte como cualquier proclamación del periodo Goulart; y el atrerido gesto final al otorgar la condecoración "Cruzeiro do Sul" al Che Guevara, justamente una semąna antes de su dimisión, fue tal vez mucho más fuerte, aunque toclavía su signifiacion precisa no está clara. En conjunto, por lo tanto, la política exterior de Goulart fue nacla más que una continuación de la de Quachros, pero si hemos de guiarnos por la campaña eleccionaria de 1960, Goulart jamás hubiese ido tan lejos si él hubiese sido quien instauraba el molde inicial. Ni Quachros ni Goulart llegaron al poder con un programa inmediato de reforma radical $y$ ninguno esturo jamás libre para actuar politicamente sin tener constantemente presente la posible resistencia de las fuerzas derechistas. Para ambos, por consiguiente, la linea indepencliente en política exterior fue sencillamente-una opción política más entre otras. Lo que demostró la camต์ กั่a de 1950 fue que, precisamente debido a la amenaza derechista, esta. opción se encontraba mucho miás fácilmente disponible para quienes, como Quadros, se manturiesen no encadenados a organización alguna de programa nacionalista.

Los méritos políticos de la línea independiente, desde la posición de Quadros, se sugieren en parte por la situación que lo confrontó en 'l Congreso al asumir el poder. El acababa de eliminar al candidato de una coalición que, a pesar de posibles desacuerdos entre sus pro. pios mienuros, estaba en general comprometida al molde de desentolrimcntismo inflacionario prevaleciente durante el periodo Kubitschek. I. lec, whes parlancntarias no roincidieron con las presidenciales, 
sin embargo, y el Congreso con el cual Quadros necesitaría trabajar -elegido en 1958 y sin posibles cambios hasta octubre de 1962- estaba dominado por la propia oposición derrotada por Quadros durante su campaña. Quaclros prometió un programa antinflacionario que necesaramente le obligaría a reducir los gastos gubernamentales $y^{\prime}$ se vió enfrentado a una codilición cle los PSD-PTB en oposición a estos compromisos y que ganaba numéricamente a la UDN (la mayor parte se cuyos miembros le apoyaban en este sentido) por 182 (116 PSD más 66 pтв) a 66. Aunque la exactitud de estas cifras exagera el verdadero estado de disciplina partidista11, decididamente Quadros tenidria que ir hacia una reorientación si cabía esperanza alguna de apoyo a su programa total. El campo de la política exterior ofrecia las mejores oportunidades en tal sentido. La situación general del país, como ya hemos visto, era tal que gran parte de la acción indispensable incluso para intereses conservadores, como una salida a los dilemas inmediatos del comercio internacional, era también deseable para la izquierda, constituyendo para ellos un paso hacia la reestructuración de la economia. Con una concesión especial a la izquiercla respecto al problema de Cuba, concesión que en todo caso aumentaba el prestigio del Brasil en otros lugares del mundo, Quadros podría posiblemente obtener una nueva coalición que pudiese estar unida, si no en cada cuestión particular por tratar, por lo menos en el senticlo de confianza general en su misión. Hacia este lin, Quadros regularmente efectuó reuniones con representantes de la mayoría de los principales bloques del Congreso, desde el vicepresidente de la UDN, Seixas Dória, al jefe del "Grupo Compacto" izquierdizante del PTB, Sérgio Magalhães; decidiendo ambos secundarlo en su politica externa También negoció con líderes del PSD, pero su éxito coń el PTB llevó al presidente del PSD, Ernani do Amaral Peixoto, a repudiar formalmente el acuerdo parlamentario PSD-PTB, basándose precisamente en la política exterior, al día siguiente de marzo 15 , fecha de presentación de la línea independiente de Quadros ante el Congreso ${ }^{13}$.

Sin embargo, ya en este momento la situación era tal que elementos del PTB, incluyenclo al secretario general del partido, Diputado Doutel de Andrade, objetaban la posición de Quadros como dañina para su propia organización. Doutel de Andrade acusaba que la política exterior independiente era un mero disfraz que encubría lo conservador cle su política interna, añacliendo que estaba especial-

"Jordan M. Young, "The Brazilian Congressional Elections", Journal of Inier-1merican Studies 5 (1963), p. 125.

LO Estado de São Paulo, marzo 16, 1961, j. 3.

4Missna fuente, marzo 17, 1961, p. 3. El discurso de Quadros se reproduce cn RBM w. it (1961), pp. 125-133. 
mente diseñada para "neutralizar" los esfuerzos hacia una organización de los grupos nacionalistast4. Este cargo fue en parte confirmado un mes más tarde cuando el Estado de São Paulo informó sobre abiertas alusiones hechas por "Janistas" sobre la conveniencia de trabajar la opción del apoyo PTB como una preparación de las elecciones de 196245 .

Pero aún si tales cargos y alusiones dan un cuadro exacto de las intenciones de Quadros, la verdad es que su táctica tuvo una lógica política propia, la que, cualesquiera fuesen sus cálculos previos, tendía a colocarlo en una posición mudho más extrema que la que sus partidarios originales podian tolerar. Asumiendo que Quadros en realidad trataba de debilitar al pro para lograr una política económica conservadora, resultaba, sin embargo, que si él quería superar a los nacionalistas en el propio campo del nacionalismo, y en forma visible para el.electorado, su defensa de Cuba en contra de las sanciones colectivas tendria que abarcar mucho más que clarificaciones privadas a los Estados Unidos. Con el transcurso del tiempo y al ver que su posición se consolidaba más y más, los partidarios conservadores de Quadros podrian haber pensado -o Quadros mismoque por muy leve que fuese el efecto de una posiciọn en política exterior sobre el bienestar inmediato de los grupos privilegiados económicamente, esta posición bien podía tener una influencia educativa, y quizás peligrosa, sobre sectores de la población hasta ahora aislados, particularmente si se la utilizaba como un sustituto de la atención a sus necesiclades materiales. Es muy probable que la renuncia de Quadros haya reflejado una conciencia de esta posibilidad -suya, de la derecha, o de ambos.

En todo caso, la secuencia rápida de hechos-condecoración a Che Guevara (agosto 19), denuncia de Quadros formulada por su antiguo aliaclo Carlos Lacercla (cadena nacional de televisión, agosto 24) 46 , y la renuncia misma (agosto 25 ) - sugiere algo de la profundidad que la división de opiniones respecto a la política exterior comenzaba a alcanzar dentro de la política brasileña. El vuelco efectuado por la derecha al abandonar a Quadros aparentemente fue repentino, sin embargo. En abril, por ejemplo, Lacerda, aunque expresó su solidaridad a los invasores de Playa Girón, seguía manteniendo su apoyo a Quadros ${ }^{47}$, a pesar de la negativa de este último

"O Estado de São Paulo, marzo 16, 1961, p. 3.

${ }^{+}$Misma fuente, abril 28, 1961 , p. 4.

"Discurso reproducido en Carlos Lacerda, O Poder das láétas (Río de Janciro, 1964), pp. 329-343. La siguiente secuencia de epiletos se aplican a la administración de Quadros: "de eretuosa, inalevolente, precaria, bohemia, desordenada, incapaz y perversa" (p. 330 ).

'O Estudo de Säo Paulo, abril 20, 1961, p. 4. 
Victor Wallis / La experiencia de Brasil con una Política Exterior Independiente

a complacer las exigencias de Estados Unidos a las sanciones colectivas en contra de Cuba ${ }^{4}$. Incluso en agosto 17, el Esiado de São Paulo, informando sobre el nombramiento del Diputado Pтв San Tiago Dantas como jefe de la delegación de Brasil ante las Naciones Uniclas, $y$ haciendo hincapié sobre la desilusión generalizada dentro del UDN respecto a" Quadros, no dejaba de recomendar por medio de un editorial que el uDN continuase apoyando el plan de acción presidencial sin hacer críticas públicas, evitando asi que otros partidos usurpasen su posición de "derecho".

Con la condecoración a Guevara, la batalla se definió claramente. Uno sólo puede interrogarse si acaso es verdad que Quadros consideró este acto, tal como lo dijeron sus defensores dentro del Congreso, un mero asunto protocolar ${ }^{49}$. Pero cualesquiera fuesen sus motivos -que éstos siguen tan misteriosos como los que llevaron a la renuncia mismano ${ }^{50}$ es muy posible que aunque Quadros hubiese continuado ejerciendo, hubiese perdido ya toda ilusión de poder actuar con una coalición derecha-izquierda falsamente aparente en un comienzo. En este sentido, el acto mismo que desencadenó la dimisión de Quadros, muy probablemente le hubiese colocado, sin dimisión, bajo inhibiciones políticas respecto a política exterior similares a las que sus opositores de coalición tuvieron que afrontar durante la campaña de 1960. Tal como estos últimos se habian sentido obligados a dișculparse por el apoyo recibido de parte del Partido Comunista, Quádros hubịese estado sujeto a presiones cada vez mayores para pedir igualmente disculpas por su supuesta simpatía hacia la Revolución Cubana.

Goulart también ejerció bajo la sombra de una constante amenaza derechista, aunque su posición fue reforzada en un cọ̣nienzo por el éxito de la resistencia armada en contra de los atentados de oficiales

\footnotetext{
4Es opinión general que ésta era la intención de la misión de Adolf A. Berle a Brasil a comienzus de marzo. Ver John Hickey, "The Day Mr. Berle Taiked with Mr. Quadros", InterAmerican Economic Alfairs 15 (verano, 1961), pp. 58-71.

$12 \mathrm{Ver}$ los debates congresionales de agosto $23 \mathrm{~g} 24$ en el Diorio. Las interpretaciones iban desde una simple condena de Quadros (por cjemplo, Diputado PSD Miguel Bahury de Maranhāo, desde p. 60+1), hasta una igual condena de Lacerda (por ejemplo, Diputado rra Eloy Dutra de Guanabara, desde p. 6070); también se opinó de que Quadros y Lacerda actuaban juntos, siendo Quadros quien planeaba su propio abandono del poder. (Diputado PTB Salvador Losacco de São Paulo, desde p. 6071). Aunque esta última teoría muestra a Quadros como a un sirviente de la derecha, una interpretación retrospcctiva, igualmente destacadora del aspecto "estratégico" de la condecoración, caracteriza a Quadros como tratando de "mostrar la unidad del proceso emancipatorio, fundiendo definitivamente sus cargas y șus éxitos" (Méendes de Almeida, n. 30, p. 60). Por otro lado, sin embargo, no debemos olvidar la ausencia de cualquicr manifestación popular organizada por Quadros para apoyarlo en los momentos de su renuncia.

onpara diferentes versiones de la renuncia, incluyenido la de Lacerda, quicn la vio como un presunto golpe de Quadros, ver Gileno de Carli, Analomia da Renincia (Rio de Janciro, 1962), Cáp. IIr. Un resumen de la versión retrospectiva del mismo Quadros aparece en $H A R$ 15 (1962), desde p. 268.
} 
militares conservadores para evitar su toma de poder. Por otro lado, muy' distinto a Quadros, él se vio impedido, debido a su dependencia del laborismo organizado, de intentar cualquiera de las medidas antinflacionaras tradicionales requeridas por la UDN. Faltándole desde un principio el apoyo conservador en forma estable e incapaz de buscar una izquierda fuerte, se vio obligado a un inmovilismo inflacionario fundado en concesiones a diestra y siniestra. Hasta enero de 1963 se vio aún más debilitado por el sistema parlamentario que le había siclo impuesto; cuando ya recobró sus completos poderes presidenciales, la situación económica era tan grave que su única esperanza era obtener una nueva dosis de empréstitos de los Estados Uniclos ${ }^{\tilde{1}}$. Como una consecuencia de todos estos factores, y sin mencionar las predilecciones personales que invitasen a la moderación a Goulart, la única medicla que unió su política exterior en modo clirecto al nacionalismo económico fue una ley limitando las remesas de utilidacles de firmas extranjerasä̃2. Esta ley aparentemente no fue puesta en vigencia, sin embargo53; y aunque así hubiese sucedido, su redacción no contenía provisión alguna sobre la distribución constructiva cle las utilidacles superfluas que quedarían dentro del pais ${ }^{54}$. En general, por lo tanto, aunque Goulart se encontró en mejor posición para mantener una línea independiente que lo que hubiese estado sin contar con el precedente Quadros; fue él tan impotente como Quadros para llevar a término Ios cambios internos esenciales a la creación de un consenso nacional que le respaldase.

Los conflictos internos de Brasil se reflejaron no sólo en el apoyo y la oposición que la política independiente despertó, sino también en su conteniclo mismo. Si acaso la influencia nacionalista era lo suficientemente fuerte para permitir que Brasil criticase a los Estados Unidos, la influencia conservadora continuó siempre igualmente fuer-

\footnotetext{
aDe ahi la misión de marzo 1963 a los Estados Unidos de San Tỉago Dantas, anterior Canciller $y$ en el momento Ministro de Finanza. Ver el discurso de Goulart durante su propia visita a Estados Unidos en abril de 1962, rcimpreso en $R B P I$ y (1962), pp. 330-335, especialmente su sugerencia de que la Alianza para el Progreso aproximase la escala del Plan Marshall en Europa (p. 533). Según Leite (Entrevista, n. 6), quien en este periodo era Director por Brasil del вin, desde la asunción del poder por Goulart, todos los eréditos provenientes de Estados Unjdos, FMI y del Banco Mundial habían sido suspendidos.

sascrita en la publicación del U.S. Department of Commerce, Overseas Business Reports 63-149 (diciembre, 1963), p. 3. Quadros habia propuesto ya una ley similar, HAR 14 (1961), p. 364. Ver también la mención hecha por Quadros de una "gradual nacionalización de utilidades" en el artículo en Forcign Allairs $\left(\mathrm{n}_{*} \cdot 15\right)$, redactado justo antes de su renursia. p, 23.

sharding (n, 27), p. 39 .

sCândido António Mendes de Almeida, Nacionalismo e Desenvolvimento (Río de Janeiro, 1963). p. 335 .
} 
Fictor Wallis / La experiencia de Brasil con una Polftica Exterior Independiente

te como para asegurar que esta crítica sólo proviniese de una "oposición leal". Esto se puede aplicar no tan sólo en relación a Cuba -donde en cierto sentido la condecoración a Guevara significó la excepción que confirma la regla- sino también, gracias en parte a la. influencia "portuguesa, en relación al Africa y al problema general de neutralidad.

Algunos de los subproductos menos polémicos del período inclependiente pueden sumariamente ser descritos. El intercambio comercial con cl bloque soviético aumentó levemente de menos del $4 \%$ hasta aproximadamente $6 \%$ del totali5, pero continuó siendo "manginal y episódico" si se considera que muy poco fue el esfuerzo desarrollado para encontrar otras complementaciones que las acostumbradas dentro del comercio brasileño existente con las naciones industrializadas $s^{50}$. Brasil estableció relaciones diplomáticas con tres de los nuevos estados africanos (Ghana, Nigeria y Senegal) y a su vez se comprometió a una cierta cantidad de becas universitarias anuales para cada uno de estos países. Además, Brasil firmó en julio de 1961 un acuerdo con la Organización Interafricana del Café, que establecía la obligación de consultas mutuas en cuanto a producción y precios ${ }^{57}$. Un gesto adicional hacia Africa fue que Brasil en agosto de 1963 se comprometió a contribuir con us $\$ 20$ millones hacia el establecimiento del Banco Africano de Desarrollons. En un nivel más amplio, Brasil participó (junto a Bolivia, Cuba y México) en la Conferencia de El Cairo de julio 1962 sobre el desarrollo económico y más tarcle publicó una crítica general a las organizaciones comerciales internacionales existentes, desde el punto de vista del mundo subdesarrollado59: posición que reiteraría clurante la Conferencia de la OEA de Ministros de Economía en noviembre de 19ồ3, en São Pautlo, y una vez más en febrero de 1964 en la reunión preparatoria de la Conferencia de Comercio y Desarrollo de Ginebra, en Alta Gracia, Argentina. Finalmente, Brasil participó como uno de los ocho estados "no comprometidos" en la Conferencia cle Desarme de Ginebra, efectuada en marzo de 1962, clestacando entre otros puntos la conveniencia del establecimiento de zonas desnuclearizadas dentro de las áreas subdesarrolladas y de recordar la necesidad de planificar

ssoverseas Business Reports 64-i5 (junio, 1964), p. 22.

onVer Mendes de Almeida (n. 54), desde p. 227, La controversia que se hubiese suscitado si el esfuerzo propuesto se hubiese cfectuado, se sugiere en la mención del poderoso Instituto de Pesquisas e Estudos Sociais a los "riesgos políticos" de cualquier dependencia en mercados comunistas, en 1pes, Reformas de Base (Rio de Janciro, 1963), p. 35.

ETRodrigucs (n. 20), desde p. 377.

es Entrevista de Leite (n. 6).

soRBPI v (1962), desde p. 561; "The Institutional Framework for the Expansion of International Trade" (publicación de la NU No a/c. 2/214, octubre 23, 1962). 
no solamente hacia el desarme e inspección, sino a su vez por la reconversión económica ${ }^{00}$.

El fundamento de la política exterior independiente, sin embargo, fue la posición asumida respecto a Cuba. El primer desafío importante que Goulart lupbo de enfrentar sobre esta determinación fue durante la Conferençia dé Cancilleres de la ofA en Punta del Este, enero de 1962, convocaḍa específicamente para considerar las sanciones colectivas. Vale la pena destacar que durante la votación decisiva sobre si efectuar o no la Conferencia, Brasil, junto a cuatro otros paises, se abstuvo, dejando que iréxico junto a Cuba fuese el único país en votar Noi1. Durante el transcurso de la conferencia misma, Brasil, representado por el Canciller de Goulart, San Tiago Dantas, se unió a México, Argentina, Bolivia, Chile y Ecuador en: a) rotar a favor de las cláusulas condenatorias del marxismo-leninismo y el actual régimen cubano como incompatibles con el sistema interamericano, pero, b) abstenerse sobre lą base de razọnes jurídicas ${ }^{02}$ en las cláusulas que expulsaban formalmeñte a Cuba de la Organización de Estados Americanos. $\mathrm{Y}$ además, junto a tres otras naçiones, Brasil se absturo de votar sobre las sanciones económicas. El discurso más. importante de San Tiago Dantas fue en parte un alegato pidiendo moderación, basándose en la convicción cle que la démocracia era capaz de triunfar sobre el comunismo en circunstancias pacíficas ${ }^{03}$; pero a su vez incluyó una reafirmación de la opinión de que el con cepto democrático no es violado esencialmente por las estructuras sociales tradicionalistas, donde, según sus palabras, la democracia sufría solamente una "pérdida momentánea"04. Brasil no alteró su posicjón oficial en ninguna ocasión posterior ${ }^{05}$. El otro gran desafío respecto a Cuba fue el presentado por la crisis de misiles de octubre de 1962. En esta oportunidad, Brasil apoyó ei bloqueo de armamentos de Estados Unidos, pero se abstuvo de participar en una moción que pedía el permiso para tomar medidas de fuerza en cuanto a los armamentos ya presentes en $\mathrm{Cuba}^{66}$. En total, por lo visto, la línea independiente de Brasil continuó siendo muy moderada incluso en

\footnotetext{
œeclaración reproducida en San Tiago Dantas, Politica Externa Independerite (Rio de Janciro, 1962), p. 214.

"La posición de México se basó en una de las exigencias del Tratado de Río de 1947 en cuanto a que una conferencia de tal naturaleza debería solamente ser convocada en situaciones de peligro inmediato. IIAR 14 (1961), p. 1143 . p. 247.

-oss, Octava Reunión de Consulta de Ministros de Relaciones Exteriores (Washington, 1963),

a fisma fuente, p. 113.

ol Misma fuente, p. 116.

"Yer, por ejemplo, el discurso ante la xu del $8 \mathrm{de}$ ícbrero, 1962, de Alfonso Arinos de Mello Franco, reproducido en $R B P I$ v (1962), pp. 370-375, especialmente la mención de la dernecracia como una mancira de ser natural de todos los paises latinoamericanos excepto Cuba (p. 372).

as Acta de! Consejo de la ora de octubre 23, 1962 (mimeo., Washington, 1962), desde p. 21.
} 
el punto clonde más molestó a Jas furrzas derechistas. Cuales fueran los proyectes contemplinless por (;oulart parn Brasil. a lo sume podia decir a Estados Uniclos que sus clesncnerdos respecto a la politica por seguir eran, tal como lo expuesto yn por San Tiago Dantas en Punta del liste, "no en cuinto a los lines... sino en cuanto a los medios'tit.

La racilación asociada con el dilema sobre Cuba nuevamente aparece (n 1:15 negociaciones brasileñas con países neutrales, En Africal, Quadros hiro sospechosas sus inicintivas dentro de los nueros estados al peclir un intercambio comercial más amplio con la República de Sudáfrica y al evitar hacer declaraciones de importancia respecto al asunto de Angolabs. La única posición fuerte tomada fut: la del Ministerio de San Tiago Dantas en enero de 1962, cuando Bra sil votó a favor de un estudio de las Naciones Unidas sobre los territorios portugueses ${ }^{60}$. Pcro, incluso entonces, el representante do Brasil ante la $\mathrm{NU}$, Alfonso Arinos de Mello Franco, excluyó todo apoyo posible a las sanciones contra cl clominio cle Portugal al exhortar a este país "a asumir la dirección del movimiento por la libertad de Angola"io. En diciembre de 1963, Brasil echó pie atrás a sus antiguas prácticas cle los años 1950 cuando sc abstuvo -junto a Estados Uniclos, Gran Bretaña y Francia, y en contraste a la posición de Venezuela- clumante una votación del Consejo de Seguridad a favor de una cláusula en que se deploraba la desobediencia del Portugal a resoluciones de la $\mathrm{Nu}$ anteriores ${ }^{i 1}$. Lejos de actuar como el líder latinoamericano del anticolonialismo, Brasil a menudo quedó rezagado en cuanto a otros países; como un cjemplo adicional sirva el de Ar-。 gentina respecto a Argelia ${ }^{\top 2}$.

En relación a los países no comprometidos como grupo, Brasil repetidamente destacó la diferencia entre su propia posición y cualquier forma de neutralismo. Quadros y San Tiago Dantas se refirieron a la política exterior independiente como una contribución al panamericanismo. San Tiago Dantas hasta la describió explícitamente como una continuación de la Operação Panamericana ${ }^{73}$ : programa

eora (n. 62), p. 110. Sobre este punto ver tambiéa la descripción general de los "abstaining countries" en John C. Dreier, The Organization of American States and the Hemisphere Crisis (New York, 1962), p. 111.

esRodrigues (n. 20), pn. 379, 385; también, "Politica Externa Independente: Um Balanço" (sin firma), Revista Cirilizaşäo Brasilrira No 1 (marzo, 1965), p. 74.

Rodrigues (n. 20), pp. 389. 425. Debe notarse que el voto sobre esta proposición fue de 99.2 (Uniled Nations Revic: 9 (1nario, 1962). p. 10).

ToDiscurso reproducido en San Tiagn Dantas (n. 59), p. 198.

it nited Nations Reticu 11 (encro, 1964), p. 19.

$=$ Rodrigues (n. 20), p. 389 .

ala mención de Quadios aparere en su artículu en Foreign Affairs (n. 15), n. 23; la decta-

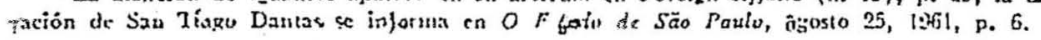

$$
\left[\begin{array}{llll}
2 & 0 & 9
\end{array}\right]
$$


que lejos de señalar hacia una fórmula de no compromiso con la guerra fría, tuvo como premisa fundamental la consideración del desarrollo económico latinoamericano como "inseparable del concepto de seguridad colectiva"it. Más a menudo, voceros de la línea independiente se limitaron a mencionar en términos muy generales las tradiciones básicamente occidentales del Brasil, tales como el cristianismo y la democracia, o, si no, simplemente indicaron que no siempre estaban de acuerdo con las posiciones tomadas por los no alineados ${ }^{\top \pi}$. Como Roberto Campos lo expresó -y a pesar de sus dudas sobre el poder del cristianismo- Brasil continuó siendo fiel a sus compromisos dentro del hemisferio occidental, y no parte de un "tercer bloque de poder", y entregado a la democracia occidental y al capitalismo ${ }^{\top 0}$. El único elemento de neutralismo que estos compromisos permitían fue el que aptamente Campos llamó "táctico". y al que describió como sencillamente una consecuencia del subdesarrollo de Brasil en relación con los Estados Unidosit.

En resumen, entonces, como los términos de la posición de Brasil en Punta del Este ya lo insinuaban, la "indepenclencia" a voces proclamada por Brasil significó primariamente la adopción de un tono de disensión denlro del sistema interamericano. Algún intento hubo de proyectar la imagen de independencia al resto del mundo, pero este intento fue más que nada verbal, como en la acusación de Araujo Castro (último Canciller de Goulart) de que el sistema interamericano no constituía un bloque, mientras que, según él, la agrupación de países neutrales -que en común tenían eỉ rechazo de la alianza con cualquiera de los dos grandes poderes- sí constituía un bloque ${ }^{78}$. Además, se puede decir que aunque los intercambios de Brasil con Africa reflejasen un creciente interés en el mundo exterior y especialmente en los problemas del desarrollo económico, esta actitud no era exclusividad suya. Brasil, siempre tratando cualquier "tercera posición" en su aspecto de bloque, se describía a sí mismo como ajeno a bloque alguno; pero en la práctica, dentro de las Naciones Unidas, nunca tomó posiciones inaceptables y continuó

"Discurso en la Üniversidad Catb́lica (n. 28), p. 142.

"Ver, por ejemplo, el discurso ante la $\mathrm{Nu}$ de septiembre 1061, de Mello Franco, en $R B P I$ IY, 16 (1961), 128, 131 .

70Roberto Campos, "Relations Between the United States and Latin America", en Mildred Adams, ed., Latin America: Etolution or Explosion? (New York, 1963), p. 37. Las afimaciones respecto al cristianismo aparecen en Campos "Sobre o Conceito de Nedtralismo", RBPI IV, 15 (1961), p. 7. Como Embajador de Goulart ante los Estados Unidos, Campos dio una visión bastante precisa de la ideología general de sua administración.

Firticulo de Campos en RBPI (n. 76), p. 9.

${ }^{8} \mathrm{De}$ ahi, por ejemplo, la negativa de Brasil para actuar de otro modo que como observador en la Conferencia de Belgrado de septiembre 1961. Alfonso Arinos de Mello Franco, Evolusão da Crise Brosileira (São Paulo, 1965), p. 254. 
rigurosamente fiel a los Estados Uniclos a trayés de todas las resoluciones relacionadas con la guerra fría, tales como la representación de China ${ }^{79}$.

Los sucesos descle el golpe militar de 1964 son lo suficientemente bien conocidos como para hacer superfluo un resumen detallado. Lo que reviste verdadera significación dentro del presente contexto es simplementc el alcance que ha adquirido la reversión de Brasil hacia moldes más tradicionales de dependencia. Sirva como indice suficiente el papel clesempeñado durante la crisis dominicana de 1965 , donde, no bastánclole un mero apoyo verbal a la intervención de los Estados Unidos, contribuyó con 1.200 soldados, incluyendo a un comandante en jefe, a las fuerzas de ocupación norteamericanas. Aunque las declaraciones más recientes de Brasil en cuanto a la política exterior indican un debilitamiento de tal entusiasmo, no alcanzan a sugerir la más mínima simpatía hacia las fuerzas contra las cuales fue dirigidoso. La "línea dura" queda no muy debajo de la superficie de la posición de gobierno.

Si uno recuerda que elementos favorables a tal política estuvieron siempre presentes durante todo el período Quadros-Goulart, las limitaciones de la linea independiente resultan'ser menos sorprendentes que sus logros. Pero hasta todo lo logrado, sin embargo, como ya he tratado de mostrar en este ensayo, bien puede ser atribuido a circunstancias particulares del período -internacionales e internas, políticas y económicas- sin suponer cambios paralelos en la composición de los intereses económicos dominantes del Brasil. Muy por el contrario, como el molde de inversiones del periodo Kubitschek lo sugiere, éstos ya habian iniciado su tendencia hacia el actual nivel de integración con los intereses norteamericanos mucho antes de la llegada al poder de Quadros. La "política exterior independiente" representaba un precario acuerdo político entre los intereses pro Estados Unidos cada vez más fuertes y sus opositores nacionales bruscamente despertados. La extrema facilidad con que el gobierno de Goulart fue depuesto sugiere, en vista de la total destrucción de los aspectos más decisivos de su línea independiente, que este acuertó jamás"se repetirá. Cualquier movimiento futuro hacia la independencia, para que obtenga un nivel comparable a lo logrado aniteriormente, requerirá cambios estructurales mucho más profundos.

PUnited Nations Revicte: (enero y novicmbre, 1962; dicicmbre, 1963).

w Véase las declaraciones del Canciller de Costa e Silva, Míagalhües Pinto, en Le Monde, 7-8 mayo, 1967 y en el cable AP del 12 de mayo 1967. 